\title{
Reciprocal Control of Drinking Behavior by Median Preoptic Neurons in Mice
}

\author{
(DStephen B. G. Abbott, ${ }^{1,2}$ Natalia L. S. Machado, ${ }^{1,3}$ Joel C. Geerling, ${ }^{1}$ and $\mathbb{C C l i f f o r d ~ B . ~ S a p e r ~}^{1}$ \\ ${ }^{1}$ Department of Neurology, Beth Israel-Deaconess Medical Center-Harvard Medical School, Boston, Massachusetts 02215, ${ }^{2} \mathrm{Heart}$ Research Institute, \\ Sydney, Australia, and ${ }^{3}$ Department of Physiology and Biophysics, Federal University of Minas Gerais, Belo Horizonte-MG 31270-901, Brazil
}

Stimulation of glutamatergic neurons in the subfornical organ drives drinking behavior, but the brain targets that mediate this response are not known. The densest target of subfornical axons is the anterior tip of the third ventricle, containing the median preoptic nucleus (MnPO) and organum vasculosum of the lamina terminalis (OVLT), a region that has also been implicated in fluid and electrolyte management. The neurochemical composition of this region is complex, containing both GABAergic and glutamatergic neurons, but the possible roles of these neurons in drinking responses have not been addressed. In mice, we show that optogenetic stimulation of glutamatergic neurons in MnP0/OVLT drives voracious water consumption, and that optogenetic stimulation of GABAergic neurons in the same region selectively reduces water consumption. Both populations of neurons have extensive projections to overlapping regions of the thalamus, hypothalamus, and hindbrain that are much more extensive than those from the subfornical organ, suggesting that the MnP0/OVLT serves as a key link in regulating drinking responses.

Key words: dehydration; lamina terminalis; optogenetics; thirst; VGAT; VGLUT2

\section{Significance Statement}

Neurons in the median preoptic nucleus ( $\mathrm{MnPO})$ and organum vasculosum of the lamina terminalis (OVLT) are known to regulate fluid/electrolyte homeostasis, but few studies have examined this issue with an appreciation for the neurochemical heterogeneity of these nuclei. Using Cre-Lox genetic targeting of Channelrhodospin-2 in transgenic mice, we demonstrate that glutamate and GABA neurons in the MnPO/OVLT reciprocally regulate water consumption. Stimulating glutamatergic MnP0/OVLT neurons induced water consumption, whereas stimulating GABAergic MnPO neurons caused a sustained and specific reduction in water consumption in dehydrated mice, the latter highlighting a heretofore unappreciated role of GABAergic MnPO neurons in thirst regulation. These observations represent an important advance in our understanding of the neural circuits involved in the regulation of fluid/electrolyte homeostasis.

\section{Introduction}

The need to consume water is fundamental for mammals to maintain fluid and electrolyte homeostasis, but the mechanisms underlying this behavior are not fully understood. The anterior wall of the third ventricle is an anatomically specialized structure that forms a continuous band of neurons that comprises the sub-

Received April 14, 2016; revised June 2, 2016; accepted June 6, 2016.

Author contributions: S.B.G.A., J.C.G., and C.B.S. designed research; S.B.G.A. performed research; S.B.G.A. and N.L.S.M. analyzed data; S.B.G.A. and C.B.S. wrote the paper.

Supported by U.S. Public Health Service (USPHS) Grants NS085477 and HL095491. S.B.G.A. is supported by an early career fellowship from the National Health and Medical Research Council of Australia (Grant GNT1052674). N.L.S.M. is supported by CNPq (National Health Council for Scientific and Technological Development) and CAPES (Coordination for the Improvement of Higher Education Personnel). J.C.G. is supported by USPHS Grants NS070682 and HL007901.

The authors declare no completing financial interests.

Correspondence should be addressed to Clifford B. Saper, Department of Neurology, Beth Israel Deaconess Medical Center, 330 Brookline Avenue, Boston, MA 02215. E-mail: csaper@bidmc.harvard.edu.

DOI:10.1523/JNEUROSCI.1244-16.2016

Copyright $\odot 2016$ the authors $\quad 0270-6474 / 16 / 368228-10 \$ 15.00 / 0$ fornical organ (SFO), the median preoptic nucleus (MnPO), and the organum vasculosum of the lamina terminalis (OVLT; Zardetto-Smith et al., 1993; Prager-Khoutorsky and Bourque, 2015). These structures are collectively considered to be essential in the regulation of body fluid and electrolyte homeostasis (Bourque, 2008; McKinley et al., 2015). The SFO is thought to be particularly important in driving angiotensin-dependent drinking behavior, as the injection of minute quantities of angiotensin II in this site elicits robust drinking responses (Simpson and Routtenberg, 1973). Stimulation of glutamatergic neurons in the SFO drives drinking behavior, while activation of GABAergic neurons reduces dehydration-induced drinking (Oka et al., 2015). However, the downstream targets of the SFO that elicit these responses are not known.

The MnPO and OVLT are major targets of SFO efferents, but the OVLT is so small that most stimulation or lesion studies probably involved the MnPO as well. Thus, although lesions of the $\mathrm{MnPO} / \mathrm{OVLT}$ region reduce drinking behavior, it is not clear 

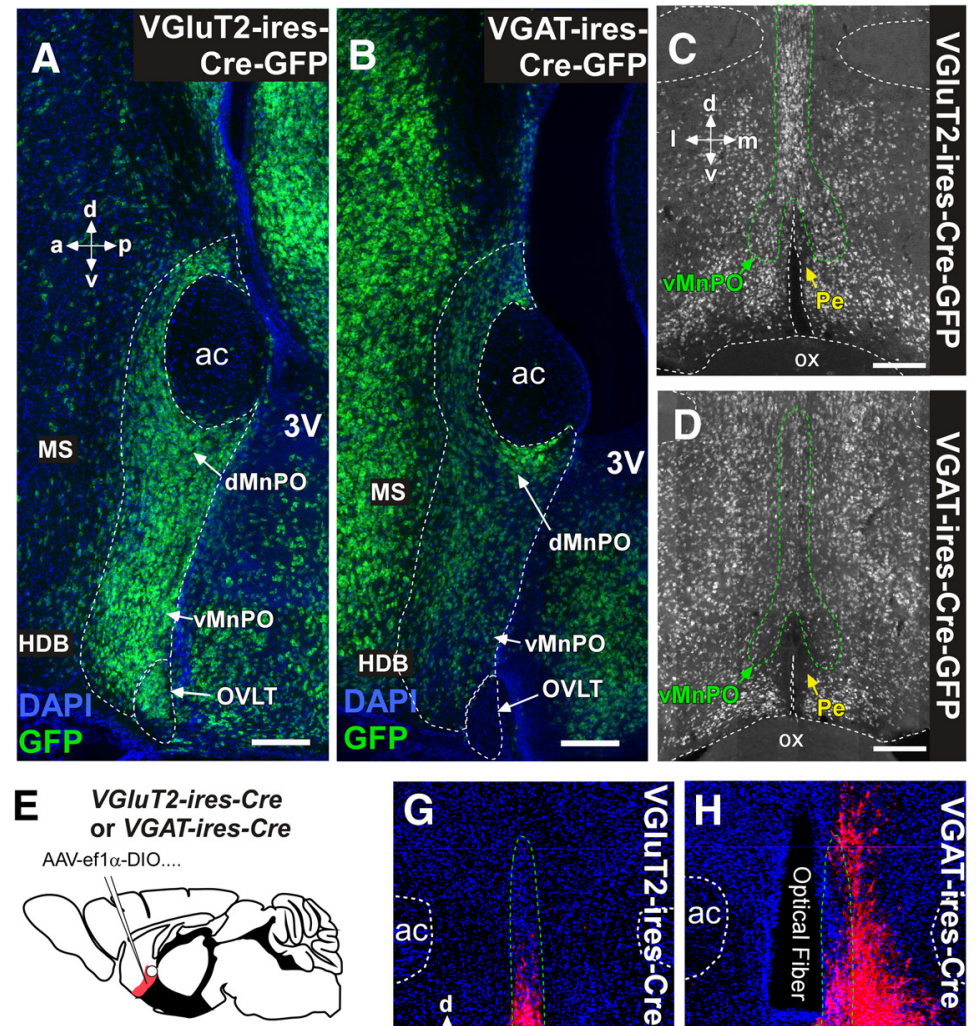

$\mathbf{F}$

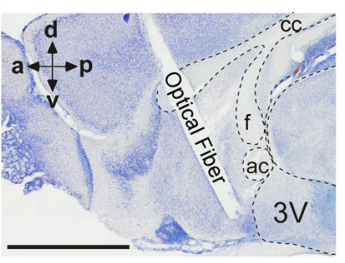

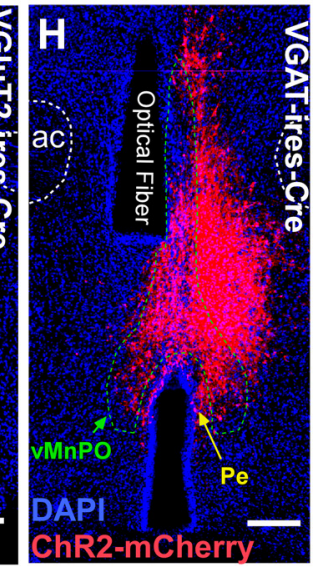

Figure 1. Distribution of VGluT2 and VGAT neurons in the POA. $A, B$, Mid-sagittal section showing the dorsal MnPO (dMnPO) and ventral MnPO (vMnPO), and OVLT in SIc17a6-cre;Rpl10-GFP (i.e., VGluT2-ires-Cre-GFP; $\boldsymbol{A}$ ) and SIc32a1-cre;Rpl10-GFP (i.e., VGAT-ires-Cre-GFP; $\boldsymbol{B}$ ) mice. $\boldsymbol{C}, \boldsymbol{D}$, Anterior POA coronal section at bregma $+0.30 \mathrm{~mm}$ showing that the density of glutamatergic neurons is higher in the MnP0 than surrounding areas, whereas the reverse is true for GABAergic neurons. $\boldsymbol{E}$, $\boldsymbol{F}$, Sagittal schematic for injections of AAV-FLEX-ChR2-mCherry showing the angle of approach $(\boldsymbol{E})$ and the tract left by optical fiber for stimulation of the MnP0/OVLT $(\boldsymbol{F}) . \boldsymbol{G}, \boldsymbol{H}$, Examples of injection sites that hit the MnP0, visualized by the expression of $m$ Cherry (native fluorescence). Note that, due to the low density of glutamatergic neurons outside the MnPO, the injection site in a VGluT2-ires-Cre ${ }^{\text {ChR2 }+}$ animal is largely confined to the MnPO, but in a VGAT-ires-Cre ${ }^{C h R 2+}$ animal, numerous cells outside the MnP0 express ChR2-mCherry. Scale bars: $\boldsymbol{A}, \boldsymbol{B}, 200 \mu \mathrm{m} ; \boldsymbol{C}, \boldsymbol{D}, 250 \mu \mathrm{m} ; \boldsymbol{F}, 1.5 \mathrm{~mm} ; \boldsymbol{G}, \boldsymbol{H}, 250 \mu \mathrm{m}$. ac, Anterior commissure; cc, corpus callosum; f, fornix; HDB, nucleus of the horizontal limb of the diagonal band; ox, optic chiasm; Pe, periventricular preoptic nucleus; $3 \mathrm{~V}$, third ventricle.

whether this is due to damage to fibers of passage from the SFO, or to cell bodies in the MnPO, OVLT, or both (Buggy and Johnson, 1977; Robertson, 1991; Cunningham et al., 1992). Together, this region has also been broadly implicated in osmoregulatory and cardiovascular regulation, as well as thermoregulation and sleep-wake regulation (Bourque, 2008; McKinley et al., 2015). However, the identity of the neurons in the OVLT or MnPO that participate in these responses have not been identified.

One challenge to understanding the role of the MnPO/OVLT in drinking behavior is that this is a complex region consisting of both GABAergic and glutamatergic neurons (Grob et al., 2003), and both excitation and inhibition of target neurons [e.g., in the magnocellular supraoptic nucleus (SON)] can be seen following electrical or chemical stimulation of the OVLT (Renaud et al., 1993; Yang et al., 1994). Osmotic stimulation of the OVLT in vitro excites vas- opressin-releasing neurons in the SON via glutamatergic-dependent mechanisms. Correspondingly, water and salt depletion following the administration of furosemide (a loop diuretic), and infusions of hypertonic saline and angiotensin II predominantly activate glutamatergic MnPO/OVLT neurons (Grob et al., 2003; Gvilia et al., 2005), which together indicate that glutamatergic MnPO/OVLT neurons are likely to drive corrective responses to dehydration, such as drinking. However, this hypothesis and the role of the GABAergic neurons in the MnPO/OVLT in drinking have not been functionally tested.

In the current experiments, we tested this hypothesis by selectively activating glutamatergic and GABAergic $\mathrm{MnPO} /$ OVLT neurons in transgenic mice using an optogenetic approach to determine their influence on water consumption. Our data provide support for these neurons, as a downstream link from the SFO, playing a key role in regulating drinking behavior.

\section{Materials and Methods}

Animals. All animal care and experimental procedures were approved by the Beth Israel Deaconess Medical Center Institutional Animal Care and Use Committee. We used male and female heterozygous Slc17a6-ires-Cre [VGluT2-ires-Cre, Slc32a-ires-cre (VGAT-iresCre; Vong et al., 2011], some of which were mated to Cre-dependent GFP reporter mice (R26-loxSTOPlox-L10-GFP; Krashes et al., 2014). Mouse lines were generously provided by B. B. Lowell (Beth Israel Deaconess Medical Center, Harvard University, Boston, MA), backcrossed to The Jackson Laboratory C57BL/6 mice, and underwent genotyping before experiments. The age of mice at the time of experimentation ranged between 12 and 30 weeks. Mice were individually housed in standard plastic cages with standard corn cob bedding with nesting materials on a $12 \mathrm{~h}$ light/dark cycle at ambient temperatures ranging between $21^{\circ} \mathrm{C}$ and $23^{\circ} \mathrm{C}$. Mouse chow (Teklad F6 Rodent Diet 8664, Envigo) and water were provided ad libitum.

Viral vectors. AAV (adeno-associated virus)DIO-ChR2 (Channelrhodospin-2; H134R)-mCherry-WPRE (woodchuck hepatitis virus post-transcriptional regulatory element; serotype 8 ; titer, $3.8 \times 10^{12}$ ), AAV-DIO-ChR2(E123/T159C)-p2a-mCherry-WPRE (serotype 5 ; titer, $4.0 \times 10^{12}$ ), and, as a control vector, AAV-DIOmCherry-WPRE (serotype 5; titer, $4.1 \times 10^{12}$ ) were produced at the University of North Carolina virus core from plasmids generously provided by K. Deisseroth (Stanford University, Palo Alto, CA).

Surgery. All surgeries were performed in sterile conditions. Mice were anesthetized with ketamine/xylazine (100 and $10 \mathrm{mg} / \mathrm{kg}$, i.p., respectively) with additional doses of $10 \%$ of the initial dose as needed to eliminate the withdrawal reflex. Stereotaxic microinjections of 15-45 nl of virus were made into the MnPO/OVLT region (coordinates: $\mathrm{AP},+1.5$ $\mathrm{mm}$; $\mathrm{L}$, midline; $\mathrm{DV},-4.4 \mathrm{~mm}$; on a $10^{\circ}$ posterior angle), and an optical fiber $(200 \mu \mathrm{m}, 0.39 \mathrm{nA})$ was implanted $300-500 \mu \mathrm{m}$ dorsal to the injection site using the same approach (Fig. 1). Age-matched littermates were 
used to create experimental and control groups by random assignment during injections of virus. Wounds were closed with surgical glue (VetBond, Santa Cruz Biotechnology), and mice were allowed to recover on a heating pad until conscious. Meloxicam, for analgesia, was administered before surgery, then again $24 \mathrm{~h}$ later. Mice were allowed to recover for $21 \mathrm{~d}$ before experimentation. Following recovery, mice showed no signs of discomfort and gained weight normally.

Measuring drinking behavior. A custom lickometer was built as per the study by Hayar et al. (2006). Junction potentials ranging from 0.5 to $1 \mathrm{~V}$ were produced when contact was made between the mouse and the water spout (internal diameter, $2.7 \mathrm{~mm}$ ), and this signal was acquired at $500 \mathrm{~Hz}$ (bandpass filter, 1-100 Hz) using Spike version 8.03 software (CED).

Visual records were acquired using a webcam (Logitech) and native software, and later were synchronized to physiological recordings using time stamps. During video scoring, we excluded drinking bouts that were $<2 \mathrm{~s}$ in duration due to the poor resolution of the onset and offset of licking. Video analysis and lickometer recordings performed in parallel demonstrate a high correlation between video scoring and spout licks $\left(r^{2}=0.80\right)$. Linear regression indicates that approximately five licks occur per second of drinking, as scored by video.

The volume of water consumption during stimulation trials was not directly measured. To estimate the volume of water consumed per spout lick, we measured bottle weight before and after a series of longer preliminary trials $(N=5$; mean licks during trials, $566 \pm 114$; mean bottle weight differential, $0.75 \pm 0.10 \mathrm{~g}$ ), which indicates that in our experimental setup mice consume $\sim 1.3 \mu \mathrm{l} /$ spout lick. This value was used to estimate water consumption during the trials presented in the text.

Experimental design. To determine water consumption caused by optogenetic stimulation of glutamatergic neurons, VGluT2-ires-Cre mice $(N=11$ in total) were individually housed in static plastic containers (diameter, $20 \mathrm{~cm}$; height, $25 \mathrm{~cm}$ ) in an insulated soundproofed recording chamber (Med Associates). Before experimentation, mice were habituated to recording conditions overnight. Bottle preference assays were performed by providing overnight access to three identical bottles containing water and two other solutions $(0.154 \mathrm{M}$ saline, and $0.4 \mathrm{M}$ saline and mineral oil), or HydroGel $\left(\right.$ Clear $\left.\mathrm{H}_{2} \mathrm{O}\right)$ placed inside the testing container. The following day, each solution or HydroGel was tested separately (single-bottle access) by performing up to $5 \times 1$ min trials, with 10-15 min separating individual trials. Two solutions were presented during two-bottle preference assays. During two-bottle assays, the bottle position was switched between trials.

Suppression of the water consumption during stimulation was measured in a disposable rodent transport cup (diameter, $15 \mathrm{~cm}$; height, 15 $\mathrm{cm}$ ) lined with a removable stainless steel mesh that was washed between trials. For this experiment, VGAT-ires-Cre mice $\left(N=8 \mathrm{ChR} 2{ }^{+}\right.$mice, $N=5 \mathrm{ChR} 2{ }^{-}$mice) were deprived of water overnight with ad libitum access to dry food in their home cage (water removed between 4:00 and 5:00 P.M.), and transferred the following morning (between 8:00 and 9:00 A.M.) to a procedure room for testing. Mice were weighed and connected to an optical fiber then were allowed to recover from handling for $15 \mathrm{~min}$ in their home cage. To ensure that the neural circuitry driving water consumption was effectively inhibited before the mice being provided access to water, the stimulation period was initiated 1-2 min before the mice being introduced to the recording chamber. A 60 min recording period was initiated upon entry to the recording chamber, and stimulation was applied during the first $30 \mathrm{~min}$ of this period. VGAT-ires-Cre mice injected with AAV-ChR2-mCherry or AAV-mCherry were examined in pairs $\left(\right.$ stim $^{+}$and stim ${ }^{-}$) in a randomized order. No food was provided in these trials. Mice were allowed to recover for at least $48 \mathrm{~h}$ before retesting.

For feeding studies, VGAT-ires-Cre mice injected with AAV-ChR2mCherry were moved to a fresh cage containing no food but ad libitum water access (between 4:00 and 5:00 P.M.) and were transferred the following morning (between 8:00 and 9:00 A.M.) to a procedure room for testing. Mice were weighed and connected to an optical fiber, then were placed in the recording chamber with no food or water access for $15 \mathrm{~min}$. Photostimulation was initiated 1-2 min before food access. Standard dry food was weighed and then added to the recording chamber at $T=0$. During the subsequent $60 \mathrm{~min}$ period, food was weighed at $T=30 \mathrm{~min}$ and $T=60 \mathrm{~min}$. No water was provided during these trials. Mice were tested in randomized pairs and were allowed to recover for at least $7 \mathrm{~d}$ after food deprivation trials before further testing.

Stimulation parameters. Photostimulation was performed with a DPSS (diode-pumped solid-state) laser (473 nM; maximum output, $100 \mathrm{~mW}$; LRS-0473-TSM-00100-10, Laserglow Technologies) controlled by Spike version 8.01 software. Stimulation of $1 \mathrm{~min}$ trains of $5 \mathrm{~ms}$ pulses at 2, 5, 10 , or $20 \mathrm{~Hz}$ was used in drinking assays in VGluT2-ires-Cre mice, and 30 min periods of phasic stimulation consisting of $20 \times 5$ ms pulses at $20 \mathrm{~Hz}$ every $3 \mathrm{~s}$ (i.e., $1 \mathrm{~s}$ on $/ 2 \mathrm{~s}$ off) were used for drinking and feeding assays in $V G A T$-ires-Cre mice. The pattern and duration of stimulation was determined empirically based on preliminary experiments (see text).

Data handling and statistical analysis. Statistical analysis was performed using Prism version 5 (GraphPad Software). Following a test for normality (D'Agostino and Pearson omnibus test or Kolmogorov- 

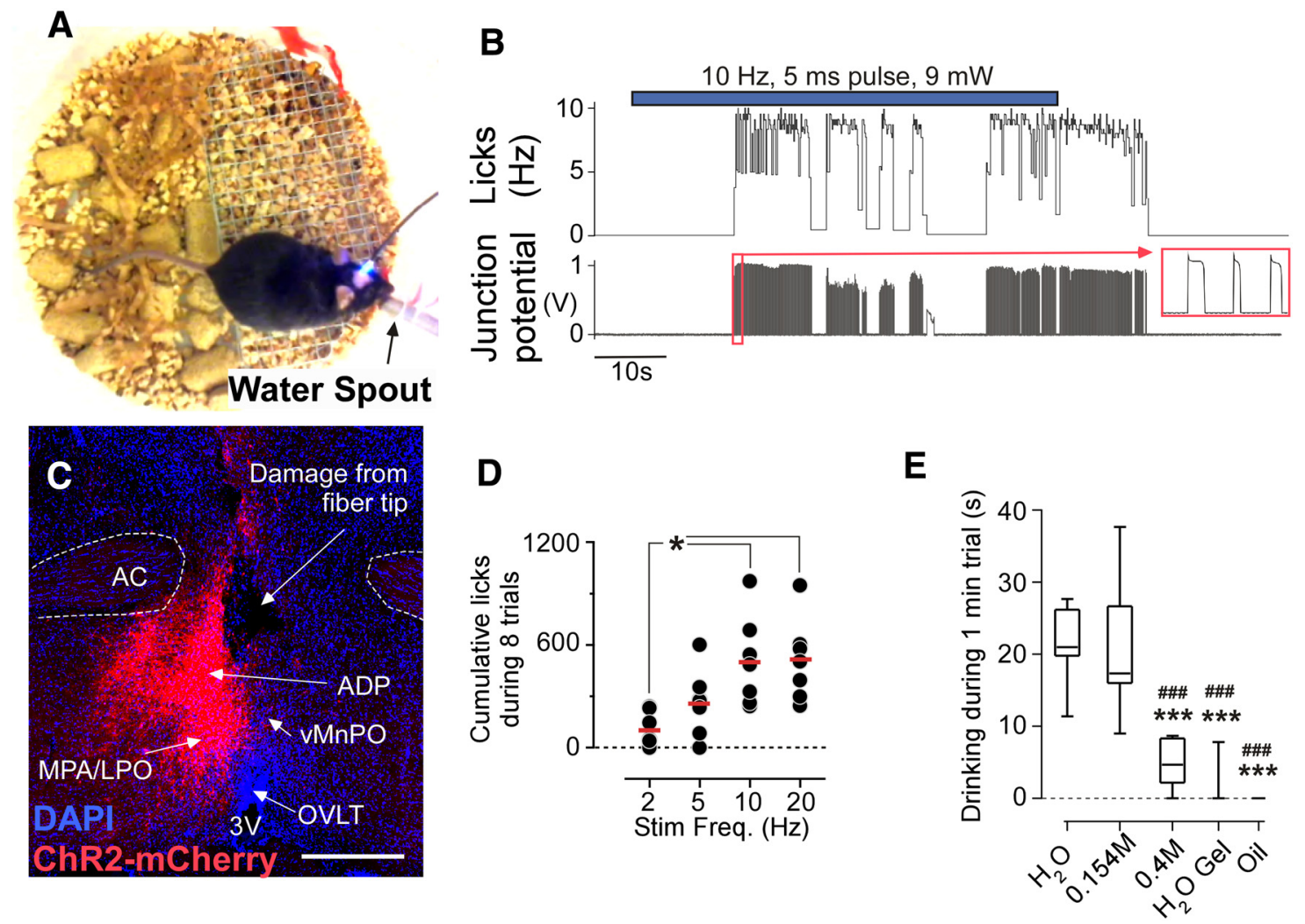

Figure 3. Stimulating excitatory neurons in the MnP0/OVLT cause water consumption. $A$, Experimental setup for testing optogenetic driving of drinking behavior. $\boldsymbol{B}$, Raw data from lickometer showing onset and pattern of drinking during $1 \mathrm{~min}$ stimulation $(10 \mathrm{~Hz}, 5 \mathrm{~ms}$ pulse, $11 \mathrm{~mW})$.C, An injection site in mouse id224 (VGluT2-ires-Cre ${ }^{\text {ChR2 }+}$ ), in which the injection was lateral to the MnPO and the animal did not consume water during stimulation. Bregma level, $+0.42 \mathrm{~mm}$. Scale bars, $250 \mu \mathrm{m}$. MPA/LPO, Medial/lateral preoptic area. $\boldsymbol{D}$, Total number of spout licks during a series of eight $1 \mathrm{~min}$ trials (i.e., $1 \mathrm{~min}$ on $/ 1 \mathrm{~min}$ off). ${ }^{*} p<0.05$. E, Solution preference assay $\left(\mathrm{N}=7 ;{ }^{* * *} p<0.001\right.$ vs $\mathrm{H}_{2} 0$, \#\#\# $<0.001$ vs saline). Box-and-whisker plot reflects range, $25 \%$ and $75 \%$ range, and median.

Smirnov normality test), significant differences were determined using either one-way or two-way ANOVA followed by a $t$ test and Bonferroni's correction (see figure legends). Sample size and power calculations (www.biomath.info) using means and SDs derived from our analysis show that our study was powered to detect an effect size of a $13 \%$ change in drinking with $>90 \%$ reliability. Data are presented as the mean \pm SEM, unless otherwise indicated.

Histology. Mice were deeply anesthetized with chloral hydrate $(1.5 \%$ body weight, i.p., 7\% solution) and transcardially perfused with $30 \mathrm{ml}$ of PBS and then $30 \mathrm{ml}$ of a $10 \%$ formalin solution. Brains were extracted and postfixed overnight in a $10 \%$ formalin solution and then stored in a $20 \%$ sucrose solution until sectioned using a freezing microtome $(35 \mu \mathrm{m}$ coronal sections into three series, or $40 \mu \mathrm{m}$ sagittal sections). Tissue was stored at $4^{\circ} \mathrm{C}$ in PBS containing the preservative sodium azide until processed for histology.

To map injection sites, one series of the preoptic area (POA) was mounted and coverslipped with HardSet mounting media containing DAPI stain (VECTASHIELD, Vector Laboratories). For immunostaining of mCherry, tissue was washed three times for 5 min in PBS, then placed in a solution containing primary antibody (rabbit polyclonal antibody raised against DSRED, a closely related red fluorescent protein, 1:10,000; lot \# 14088015, catalog \#632496, Clontech), 2.5\% Triton $\mathrm{X}-100$, and $1 \%$ normal horse serum (NHS) in PBS. The next day, tissue was rinsed five times over $30 \mathrm{~min}$ in PBS, then was incubated for $1-2 \mathrm{~h}$ in secondary antibody (1:1000; biotin-SP-conjugated donkey $\alpha$ rabbit IgG; lot \#121173, code catalog \#711-065-152, Jackson ImmunoResearch), $2.5 \%$ Triton X-100, and $1 \%$ NHS in PBS. Following a series of five washes, tissue was then incubated in $A B C$ solution (1:1000) for $1 \mathrm{~h}$, rinsed again, and then stained using a DAB reaction. In these cases, tissue was mounted, Nissl counterstained, dehydrated, and mounted of xylene with Permamount. Staining of sections from brains without injections of mCherry-expressing AAV produced no labeling.
Mapping of injection site. Neurons transduced with ChR2-mCherry or ChR2-p2a-mCherry, and locations of the fiber optic tip were plotted and interpolated onto standardized coronal sections separated by $120 \mu \mathrm{m}$ (Paxinos and Franklin, 2012). Plotted injection sites captured all transduced neurons, regardless of cell density. For presentation purposes, injections sites were overlaid, and the opacity of the fill color for each mapped injection site was adjusted so that complete overlap of all cases in each dataset yielded a solid/nontransparent color.

\section{Results}

The MnPO/OVLT in mice is composed of both vesicular glutamate transporter 2 and vesicular GABA transporter neurons

We first evaluated the distribution of glutamatergic and GABAergic neurons in the MnPO/OVLT of mice by crossing GFP reporter mice with VGluT2-ires-Cre and VGAT-ires-Cre mice. Vesicular glutamate transporter 2 (VGluT2) neurons were densely packed in the MnPO and OVLT in the midline region, but were relatively sparse in the adjoining preoptic and forebrain regions (Fig. $1 A, C$ ), whereas vesicular GABA transporter (VGAT) neurons were numerous and evenly distributed throughout $\mathrm{MnPO}$ and adjacent preoptic and anterior forebrain regions, but were sparse in the OVLT (Fig. $1 B, D$ ). These expression patterns are consistent with the expression of GAD65, GAD67, and VGluT2 mRNA in the rat (Grob et al., 2003) and mouse (http://mouse.brain-map.org, see experiments 71724696, 479, and 79591669).

To determine the role of MnPO/OVLT VGluT2 and VGAT neurons in water consumption, we injected an AAV conferring Cre-dependent expression of Channerlrhodopsin2-mCherry or a control AAV expressing Cre-dependent mCherry in VGluT2- 

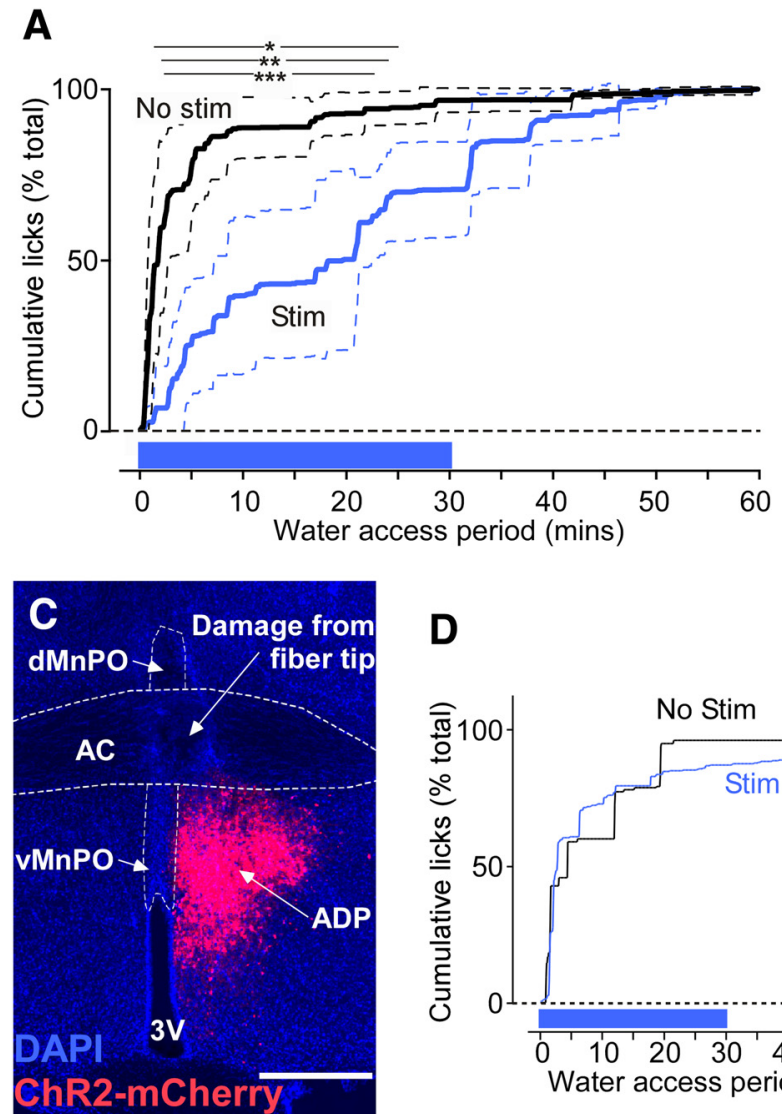

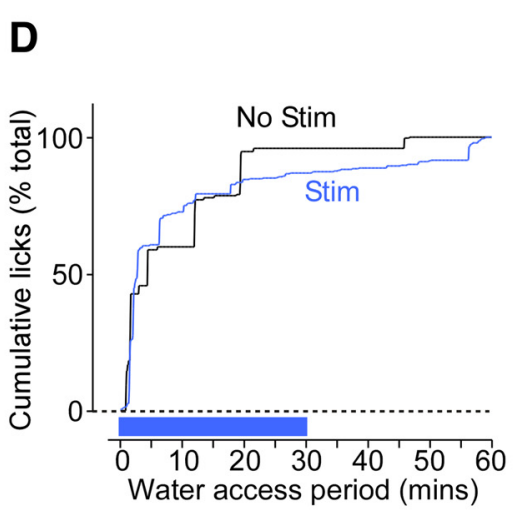

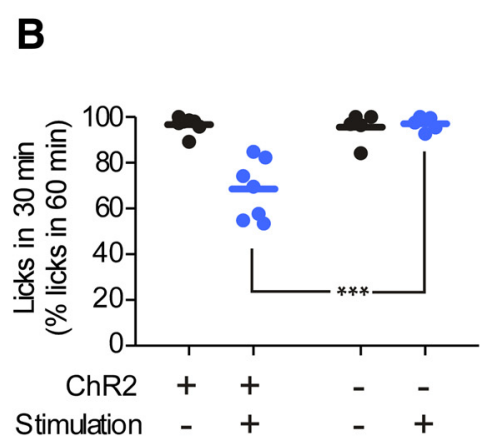

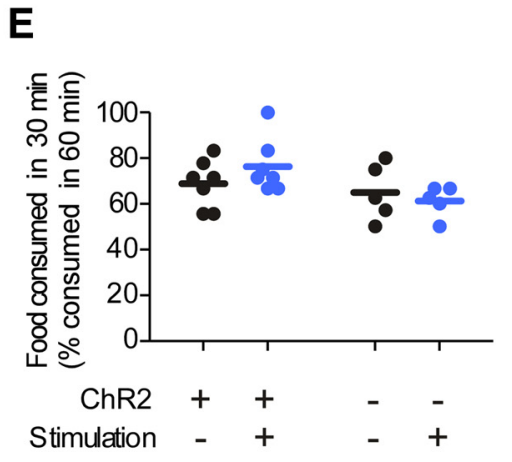

Figure 4. Stimulating inhibitory neurons in the MnP0/OVLT reduces water consumption, but not food consumption. $A$, Normalized cumulative spout licks (mean \pm SD, $N=7$ ) during 60 min of water access in water-deprived VGAT-ires-cre ${ }^{\text {ChR2 }}+$ mice. Stimulation ( $1 \mathrm{~s} \mathrm{on} / 2 \mathrm{~s}$ off, $20 \mathrm{~Hz}, 5 \mathrm{~ms}, 13 \mathrm{~mW}$; in blue) was applied for the first 30 min of the water access period. ${ }^{*} p<0.05,{ }^{* *} p<0.01$, ${ }^{* * *} p<0.001$ by post hoc $t$ test with Bonferroni's correction. $\boldsymbol{B}$, Normalized spout licks by water-deprived mice during the first 30 min of the 60 min access period. ${ }^{* * *} p<0.001$ by post hoc $t$ test with Bonferroni's correction). $C$, Injection site in a VGAT-ires-Cre ${ }^{\text {ChR2 }}$ mouse in which the injection was lateral to the MnP0, and in which the animal had no response to optogenetic stimulation during water repletion after dehydration. Bregma level, $-0.12 \mathrm{~mm}$. Scale bars, $250 \mu \mathrm{m}$. D, Time course of cumulative water spout licks during a $1 \mathrm{~h}$ access period following overnight water deprivation for the mouse shown in $\boldsymbol{C}$. $\boldsymbol{E}$, Food consumed by food-deprived mice during the first $30 \mathrm{~min}$ of food access as a percentage of the total amount of food consumed in 60 min.

ires-Cre and VGAT-ires-Cre mice and implanted an optical fiber anterodorsally to the MnPO for light delivery (Fig. $1 E, F$ ). In

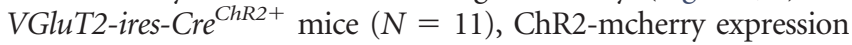
was observed predominantly in neurons in the ventral $\mathrm{MnPO}$, OVLT, and occasionally the dorsal periventricular regions of the POA (Figs. 1G, 2B), the anterodorsal preoptic nucleus (ADP), and rarely in the medial septum (MS) and diagonal band of Broca (DBB). In VGAT-ires-Cre ${ }^{C h R 2+}$ mice $(N=7)$, transduced neurons were predominantly located in the $\mathrm{MnPO}$, owing to the scarcity of VGAT $^{+}$neurons in the OVLT; labeled neurons were also found to varying degrees in the $\mathrm{ADP}, \mathrm{DBB}$, and MS (Figs. $1 \mathrm{H}, 2 \mathrm{D}$ ). The rostrodorsal tip of the MnPO was rarely transduced in either mouse strain. Also, because we used an angled anterior approach for our injections and fiber implantation (Fig. $1 F$ ), the SFO was not damaged and did not contain neurons expressing ChR2-mcherry in either strain of mouse.

\section{Activating VGluT2 MnPO/OVLT neurons drives water consumption}

Photostimulation of MnPO/OVLT VGluT2 neurons in fully satiated VGluT2-ires-Cre $e^{C h R 2+}$ mice $(N=7)$ produced immediate and vigorous consumption of water (Fig. $3 A, B$ ). The latency from the onset of stimulation to the first drinking bout ranged between 4 and $38 \mathrm{~s}$ (median, $17 \mathrm{~s}$ ), and drinking would sometimes continue beyond the end of the stimulation period (Fig. $3 B$ ). Water consumption was never observed during photo- stimulation in VGluT2-ires-Cre $e^{C h R 2+}$ mice $(N=2)$ in which injections of virus were placed lateral to the MnPO/OVLT (Fig. $3 C)$, or in mice injected with a control virus $(N=7$; Fig. $2 A)$. Food consumption was not observed during periods of stimulation in VGluT2-ires-Cre $e^{C h R 2+}$ or in control mice. In VGluT2ires-Cre $\mathrm{ChR2+}^{-}$mice, the intensity of water consumption was dependent on stimulation frequency with an apparent ceiling at $\sim 10 \mathrm{~Hz}$ (Fig. 3D). Over a series of eight sequential trials at $10 \mathrm{~Hz}$ over $15 \mathrm{~min}$, we observed $502 \pm 99$ licks (Fig. 3D), which is equivalent to a mean water intake of $0.65 \pm 0.14 \mathrm{ml}$. During 1 min of photostimulation in a single-bottle test, VGluT2-ires$\mathrm{Cr} e^{\mathrm{ChR2+}}$ mice $(\mathrm{N}=7)$ displayed a strong preference for drinking water and physiological saline solution $(21 \pm 2$ and $20 \pm 4 \mathrm{~s}$, respectively) relative to a high-salt solution $(0.4 \mathrm{M} ; 4 \pm 1 \mathrm{~s})$, gelatinized water, and mineral oil (repeated measures one-way ANOVA: $F_{(1,6)}=28.57, p<0.0001$; Fig. $3 E$ ). In a two-bottle preference test, mice $(N=5)$ showed no preference for saline solution over water $\left(13 \pm 5\right.$ vs $10 \pm 3 \mathrm{~s} ; N=5$; paired $t$ test: $t_{(4)}=$ $0.73, p=0.50)$ and a clear preference for water over a high-salt solution $\left(23 \pm 2\right.$ vs $1 \pm 1 \mathrm{~s}$; paired $t$ test, $\left.t_{(4)}=8.58, p=0.001\right)$.

Activating VGAT MnPO/OVLT neurons attenuates water consumption in dehydrated mice, but not food consumption in food-deprived mice

Photostimulation of MnPO/OVLT VGAT neurons had no observable effect in water-satiated mice $(N=7)$, so we evaluated the 
Table 1. Qualitative summary of the density of terminals for VGLUT2 and VGAT MnPO/OVLT neurons based on conditional tracing of cases demonstrating robust physiological responses

\begin{tabular}{|c|c|c|c|c|c|}
\hline & $\begin{array}{l}\text { VGluT2-ires-Cre } \\
\text { mice }\end{array}$ & $\begin{array}{l}\text { VGAT-ires-Cre } \\
\text { mice }\end{array}$ & & $\begin{array}{l}\text { VGluT2-ires-Cre } \\
\text { mice }\end{array}$ & $\begin{array}{l}\text { VGAT-ires-Cre } \\
\text { mice }\end{array}$ \\
\hline Commissural nucleus of the solitary tract/central canal & ++ & + & Dorsomedial hypothalamus (dorsal) & +++ & +++ \\
\hline Raphe obscurus & +++ & + & Perifornical area & +++ & +++ \\
\hline Parapyrimidal region & +++ & + & Lateral hypothalamus & +++ & +++ \\
\hline Raphe pallidus & +++ & + & Median eminence & +++ & + \\
\hline Barrington's nucleus/laterodorsal tegmental nucleus & +++ & +++ & Lateroanterior hypothalamic nucleus & +++ & ++ \\
\hline Locus coeruleus & ++ & ++ & Paraventricular nucleus of the thalamus & +++ & ++ \\
\hline Lateral parabrachial nucleus region & +++ & ++ & Paraventricular hypothalamic nucleus (posterior) & +++ & +++ \\
\hline Dorsal raphe/median raphe & + & +++ & Paraventricular hypothalamic nucleus (parvocellular) & ++ & ++ \\
\hline Ventrolateral periaqueductal gray & +++ & +++ & Paraventricular hypothalamic nucleus (magnocellular) & +++ & +++ \\
\hline Pedunculopontine tegmental nucleus & ++ & ++ & Periventricular hypothalamic nucleus & +++ & +++ \\
\hline Dorsal and dorsolateral periaqueductal gray & ++ & + & Xiphoid thalamic nucleus & +++ & ++ \\
\hline Tuberomammillary nucleus & ++ & ++ & Reuniens thalamic nucleus & ++ & ++ \\
\hline Premammillary nucleus & ++ & ++ & Supraoptic nucleus & +++ & ++ \\
\hline Arcuate nucleus & +++ & +++ & Subfornical organ & ++ & +++ \\
\hline Dorsomedial hypothalamus (ventral) & +++ & +++ & Dorsolateral preoptic nucleus & +++ & +++ \\
\hline Dorsomedial hypothalamus (compact) & ++ & ++ & & & \\
\hline
\end{tabular}

+ , Sparse terminal field; ++ , moderate terminal field; +++ , dense terminal field.

effect of stimulation in water-deprived mice. During $60 \mathrm{~min}$ of water access after overnight deprivation, unstimulated VGAT-ires-Cre $\mathrm{ChR2+}^{\mathrm{mice}}$ avidly consumed water in the first 30 min of access to $96 \pm 1 \%$ of their total intake in $1 \mathrm{~h}$ (Fig. $4 A, B$ ). In trials in which MnPO/OVLT VGAT neurons were stimulated during the first $30 \mathrm{~min}$ of water availability, mice consumed only $68 \pm 5 \%\left(\mathrm{CHR}^{+}{ }^{+}\right.$stim $^{-}{ }^{-}$vs stim ${ }^{+}$, paired $t$ test: $t_{(6)}=5.014, p=$ 0.0024 ) of their total intake in the first $30 \mathrm{~min}$ of the $60 \mathrm{~min}$ access period (Fig. $4 A, B$ ), though the total number of licks in $60 \mathrm{~min}$ was similar for both conditions $(331 \pm 67$ vs $339 \pm 58$ licks; paired $t$ test: $\left.t_{(6)}=0.1, p=0.92\right)$. Stimulation in water-deprived VGAT-ires-Cre mice injected with a control virus had no effect on water consumption ( $96 \pm 3 \%$ vs $97 \pm 1 \%$; $N=5$; Fig. $4 B$ ),

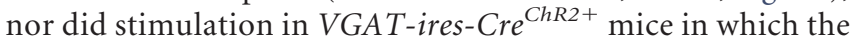
ChR2-mCherry expression was not within the MnPO/OVLT (Fig. 4C,D). Importantly, photostimulation of the $\mathrm{MnPO} /$ OVLT in VGAT-ires-Cre $e^{C h R 2+}$ had no effect on food consumption following food deprivation $(69 \pm 4 \%$ vs $76 \pm 4 \%$ in the first $30 \mathrm{~min}$ of a $60 \mathrm{~min}$ access period; paired $t$ test: $t_{(6)}=$ 1.556, $p=0.1708$; Fig. $4 E)$.

\section{Brain regions targeted by ChR2-expressing MnPO/OVLT VGLUT2 and VGAT neurons}

These last experiments examined the CNS regions that most plausibly modulated water consumption and water/electrolyte homeostasis in response to VGAT and VGLUT2 neuron stimulation. For this analysis, we examine the terminal distribution of CHR2-mCherry-labeled axons originating from injection sites that were largely restricted to the MnPO/OVLT and yielded robust physiological responses during stimulation experiments. For comparison, we also examined cases in which transduced neurons were absent from the MnPO/OVLT and did not yield a detectable physiological response.

In general, the fibers and boutons arising from both VGAT and VGLUT2 neurons in the MnPO/OVLT overlapped extensively and innervated brain regions that largely recapitulated the observations in the rat using classic tracing methods (Saper and Levisohn, 1983; Uschakov et al., 2007). The approximate density of VGAT versus VGLUT2 innervation varied between targets and is summarized in the Table 1 . As has been described in detail elsewhere, fibers exited the MnPO/OVLT in the following two primary pathways: first, via a midline pathway within the periventricular tissue of the third ventricle; and, second, via a laterally and ventrally sweeping fiber tract that travels through the lateral hypothalamic area. These pathways converge in the ventral midline of the pontine-hypothalamic junction before ascending into the central gray matter, with a small group of fibers passing through the ventral raphe nuclei en route to the caudal brainstem. In addition, both populations of neurons in the MnPO/OVLT had collaterals within the SFO and dorsal MnPO.

Glutamatergic and GABAergic MnPO/OVLT neurons had fibers and terminals in the lateral hypothalamus, periaqueductal gray (predominantly within the ventrolateral and lateral gray matter), locus ceruleus, tuberomammillary nucleus, mediodorsal and paraventricular thalamic nucleus, nucleus reuniens, and lateral habenula (Fig. 5A-J). These regions underlie processes related to arousal, decision-making, and affect. Labeled fibers were observed bilaterally in the amygdaloid complex; entorhinal, perirhinal, and piriform cortices; and hippocampus in mice of both genotypes with injections sites incorporating the MS and $\mathrm{DBB}$, but these fibers were absent from cases where injection sites avoided these regions. As mentioned already, fibers of both neuronal populations were observed in the SFO, though the inhibitory innervation appeared denser (Fig. 6A,B), which may contribute to the inhibition of drinking during GABAergic neuron stimulation. Consistent with the important role of the $\mathrm{MnPO} / \mathrm{OVLT}$ in the neuroendocrine response to perturbations in water/electrolyte homeostasis, the SON, including its retrochiasmatic part, was heavily innervated by both glutamatergic and GABAergic MnPO/OVLT neurons, as were the magnocellular neurons in the paraventricular hypothalamic nucleus (PVH; Fig. $6 C-H)$. Finally, MnPO/OVLT VGLUT2 and VGAT neurons innervated brain regions that contribute to the autonomic response to perturbations in water/electrolyte homeostasis, including the parvicellular PVH, dorsomedial hypothalamus, dorsal hypothalamic area, pontine gray matter, raphe pallidus (Fig. $5 K, L$ ), and raphe obscurus.

In summary, these two pathways, with presumably opposite postsynaptic effects and opposite effects on drinking, target almost all of the same structures, indicating that they likely constitute a "push-pull" mechanism that can switch drinking on or off. 

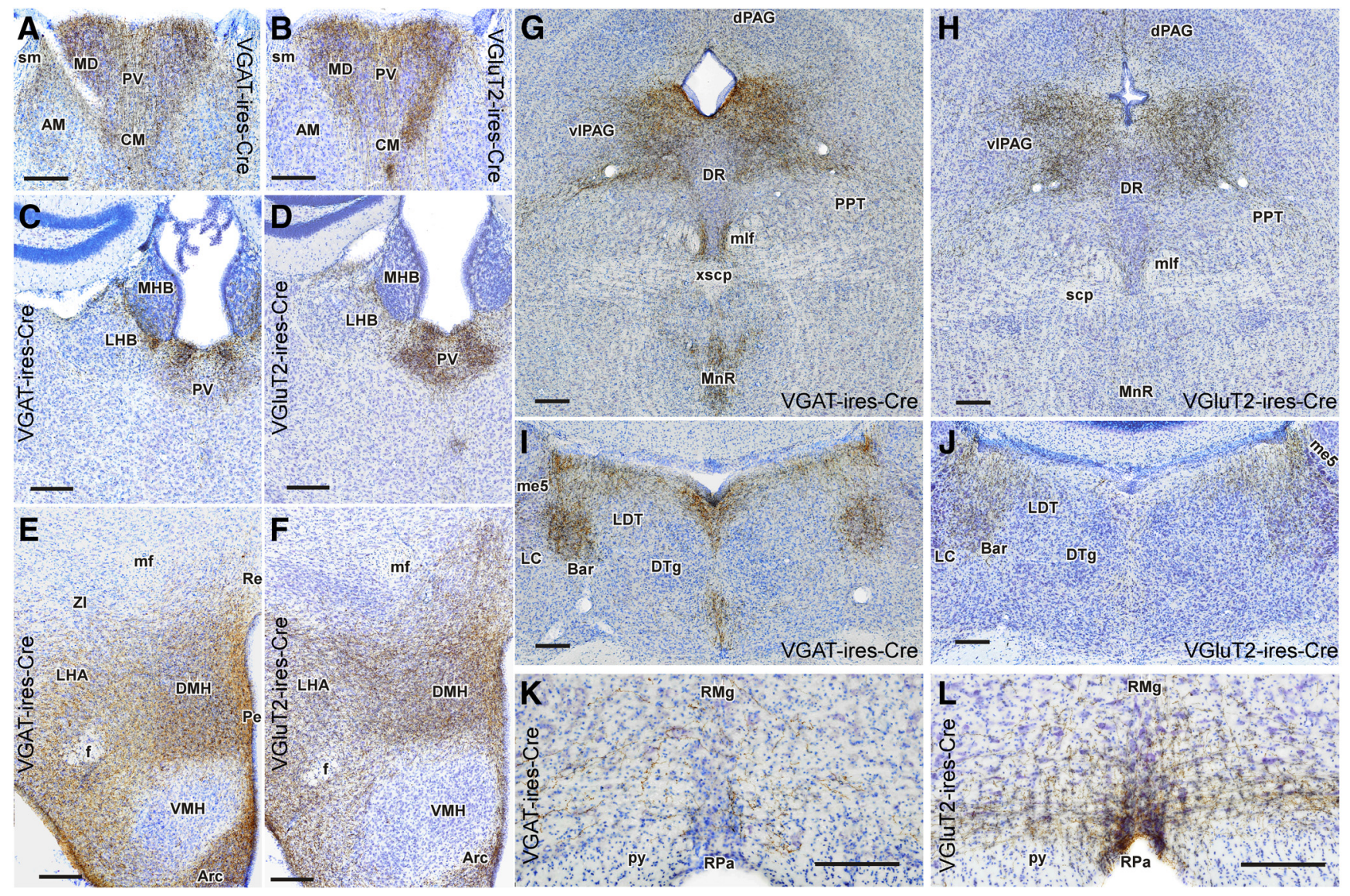

Figure 5. Projections of VGluT2 and VGAT neurons. $\boldsymbol{A}-\boldsymbol{J}$, Glutamatergic and GABAergic MnPO/OVLT neurons innervate the paraventricular thalamic nucleus $(\boldsymbol{A}-\boldsymbol{D})$; the lateral habenula $(\boldsymbol{C}, \boldsymbol{D})$; the tuberal hypothalamus $(\boldsymbol{E}, \boldsymbol{F})$; the periaqueductal gray matter, raphe nuclei, and pedunculopontine tegmental region $(\boldsymbol{G}, \boldsymbol{H})$; laterodorsal tegmental nucleus, locus ceruleus, and Barrington's nucleus $(\boldsymbol{I}, \boldsymbol{J})$; and the medullary raphe $(\boldsymbol{K}, \boldsymbol{L})$. Injection sites for each respective case are shown in Figure $1, G$ and $H$. Scale bar, $200 \mu \mathrm{m}$. AM, Anterodorsal thalamic nucleus; Arc, arcuate nucleus; Bar, Barrington's nucleus; dPAG, dorsal periaqueductal gray; DMH, dorsomedial hypothalamus; DR, dorsal raphe; DTg, dorsal tegmentum; f, fornix; me5, mesenphalic nucleus 5; MHB, medial habenula; mlf, medial longitudinal fasciculus; MnR, median raphe; PPT, pedunculopontine tegmental nucleus; LC, locus coeruleus; LDT, lateral dorsal tegmentum; LHA, lateral hypothalamic area; LHB, lateral habenula; Pe, periventricular area; PV, paraventricular nucleus of the thalamus; py, pyramidal tract; Re, reuniens thalamic nucleus; RMg, raphe magnus; RPa, raphe pallidus; vIPAG, ventrolateral periaqueductal gray; VMH, ventromedial hypothalamus; xscp, decussation of the superior cerebellar peduncle; Zl, zona incerta.

Our optogenetic stimulation studies show that this switch operates on a timescale of seconds, suggesting that it powerfully and directly drives or inhibits the behavior, rather than acting through intermediate mechanisms, such as a dry mouth signaling thirst.

\section{Discussion}

Drinking is an innate behavior that is tightly regulated to maintain fluid/electrolyte homeostasis. In this study, we demonstrated that the MnPO/OVLT in mice are composed of a mixture of glutamatergic and GABAergic neurons, with GABAergic neurons mainly in the $\mathrm{MnPO}$, and that selective optogenetic stimulation of the glutamatergic neurons robustly drives water intake, whereas stimulation of the GABAergic neurons suppresses drinking. Despite these diametrically opposed functions, conditional mapping of the axons and terminal fields of glutamatergic and GABAergic MnPO/OVLT neurons reveals that they essentially innervate the same targets. Thus, the MnPO/OVLT is a neural substrate for the bidirectional control of drinking behavior. This potent behavioral control must be considered in the context of other evidence that the MnPO/OVLT is involved in salt appetite, and the control of vasopressin and renin secretion and renal sodium secretion; in other words, that this is a key integrative site for behavioral, endocrine, and autonomic control of fluid and electrolyte balance.
Several considerations are important in the interpretation of the data. First, our injections invariably encroached upon structures adjacent to the MnPO/OVLT, and we cannot exclude the possibility that these regions also influence drinking behavior. Specifically, there is evidence that neurons in the vertical limb of the DBB inhibit vasopressin secreting SON neurons in response to stimulating the arterial baroreceptors (Renaud et al., 1993), and neurons in this vicinity expressed CHR2-mcherry in six of seven VGAT-ires-Cre $e^{C h R 2+}$ cases that displayed an inhibition of thirst. However, the earlier studies may also have involved parts of the $\mathrm{MnPO}$, which is adjacent to the DBB. Indeed, effective responses to cell-specific stimulation (i.e., inhibition or excitation of water consumption without involving fibers of passage) were observed only in cases that had substantial ChR2-mcherry expression in the MnPO/OVLT, and placement of the optical fiber anterior and dorsal to the MnPO/OVLT. Thus, we believe that the stimulation of MnPO/OVLT neurons is the primary cause of the observed effects. Our anatomical observations also suffer from the inability to produce cases with ChR2-mcherryexpressing neurons exclusively in the MnPO/OVLT, particular in VGAT-ires-Cre $\mathrm{ChR2+}^{+}$mice. Thus, additional retrograde tracing experiments will be useful in confirming the involvement and neurochemical content of neurons in the MnPO/OVLT in ac- 

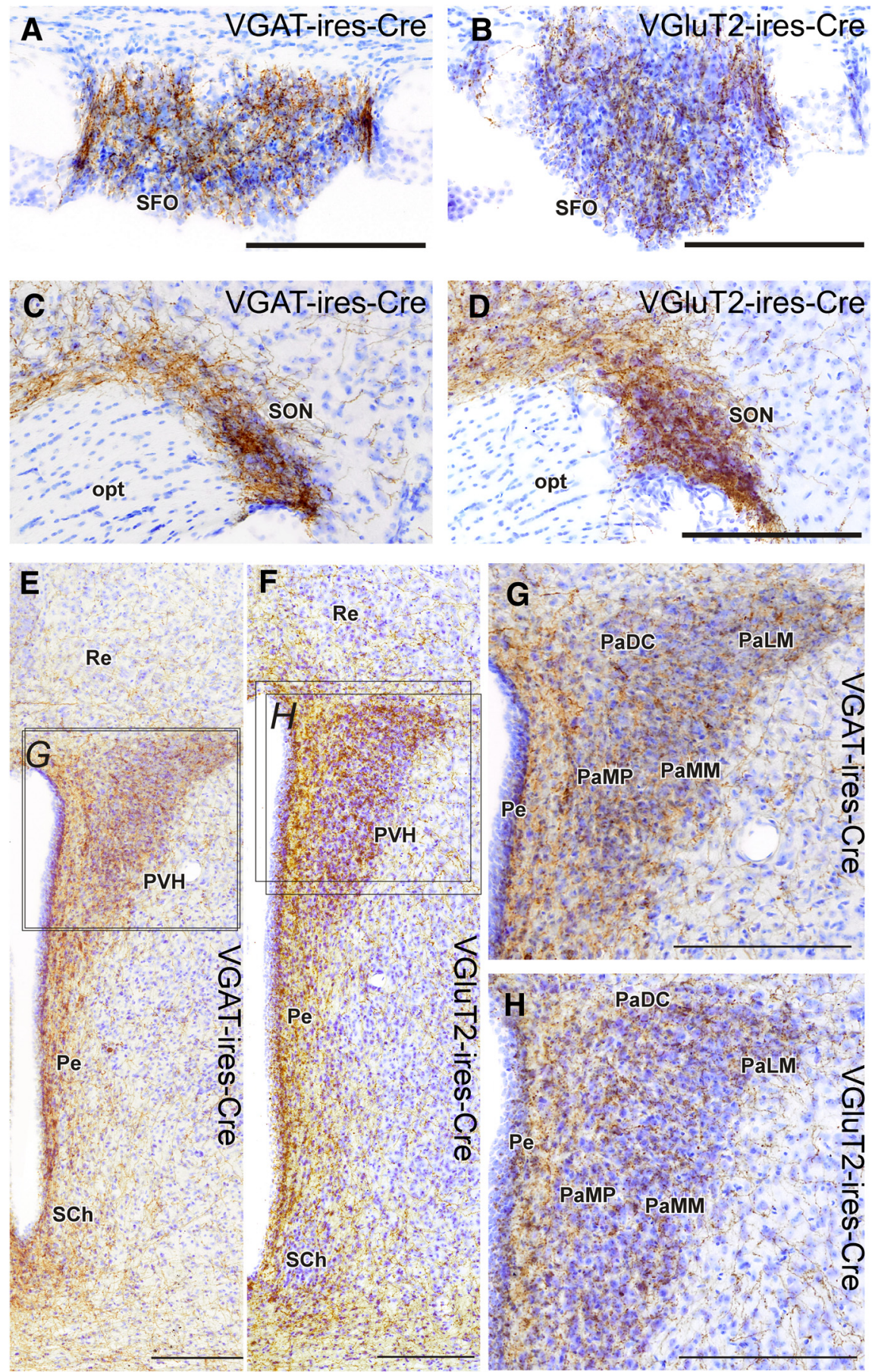

Figure 6. Projections to MnP0/OVLT VGluT2 and VGAT neurons in brain regions involved in neuroendocrine response to dehydration. $\boldsymbol{A}-\boldsymbol{H}$, Glutamatergic and GABAergic MnPO/OVLT neurons innervate the subfornical organ $(\boldsymbol{A}, \boldsymbol{B})$ supraoptic nucleus $(\boldsymbol{C}, \boldsymbol{D})$, and paraventricular nucleus of the hypothalamus $(\boldsymbol{E}-\boldsymbol{H})$. Injection sites for each respective case are shown in Figure $1, G$ and $H$. Scale bar, $200 \mu \mathrm{m}$. opt, Optic tract; $P$ aDC, paraventricular hypothalamic nucleus (dorsal cap); PaLM, paraventricular hypothalamic nucleus (lateral magnocellular); PaMM, paraventricular hypothalamic nucleus (medial magnocellular); PaMP, paraventricular hypothalamic nucleus (medial parvicellular); Pe, periventricular hypothalamic nucleus; Re, reuniens thalamic nucleus; $\mathrm{SCh}$, suprachiasmatic nucleus.

tivating brain regions that are important in water consumption.

Second, the anatomical proximity of MnPO and OVLT neurons prevented us from establishing a clear functional distinction between these two populations. However, the OVLT contains very few GABAergic neurons, so the inhibition of drinking is likely to be mediated almost entirely by the MnPO. On the other hand, the most robust drinking responses during stimulation were observed in cases with ChR2-mcherry incorporated into both structures.

Finally, although our results focused on the role of glutamatergic and GABA ergic neurons in the MnPO/OVLT in water consumption, GABAergic and nonGABAergic (presumably glutamatergic) neurons in the MnPO/OVLT have been implicated in other functions, such as thermoregulation (including fever responses) and sleep-wake control (Lazarus et al., 2007; Nakamura and Morrison, 2008; Szymusiak and McGinty, 2008). It is likely that some of the targets we have identified for MnPO/OVLT axons relate to these other functions. It is not known to what extent individual MnPO/OVLT neurons participate in several of these functions, whether they are controlled by separate populations of intermixed neurons, or whether neurons involved in specific functions (or combinations of functions) express unique genetic signatures that can be used to further subdivide and manipulate these neurons.

Numerous in vivo rodents studies have demonstrated that neurons in $\mathrm{MnPO} /$ OVLT are activated by stimuli associated with dehydration (hyperosmotic and hypovolemic stimuli, and elevated circulating angiotensin; Oldfield et al., 1991; McKinley et al., 1994, 2008; Thunhorst et al., 1998; De Luca et al., 2002; Stocker and Toney, 2005; Taylor et al., 2008; Kinsman et al., 2014). Glutamatergic MnPO/OVLT neurons appear to be predominantly activated during these stimuli (Grob et al., 2003; Gvilia et al., 2005), but until now no study has examined the functional role of glutamatergic MNPO/OVLT neurons in water consumption. We demonstrate that the activation of glutamatergic $\mathrm{MNPO} /$ OVLT neurons causes immediate and voracious water consumption in waterreplete mice. Furthermore, the heavy innervation of the SON, magnocellular $\mathrm{PVH}$, and regions important in autonomic control, such as the dorsomedial hypothalamus, parvocellular $\mathrm{PVH}$, and medullary raphe, suggest that activating glutamatergic MNPO/OVLT neurons also causes vasopressin and/or oxytocin release and sympathetic activation. These findings suggest that the attenuation of water consumption and vasopressin release following $\mathrm{MnPO} / \mathrm{OVLT}$ lesions or inactivation in previous reports (Buggy and Johnson, 1977; Thrasher et al., 1982; Mangiapane et al., 1983; Cunningham et al., 1991, 1992; Robertson, 1991) was caused by the destruction or inhibition of glutamatergic $\mathrm{MnPO} / \mathrm{OVLT}$ neurons. They also suggest that the release of $\mathrm{MnPO} / \mathrm{OVLT}$ targets from inhibition is not sufficient to drive a drinking or vasopressin response. 
However, our demonstration that selectively activating MnPO GABAergic neurons reduces water consumption in dehydrated mice provides strong evidence that some subpopulations of $\mathrm{MnPO}$ neurons can potently suppress drinking. Importantly, the inhibition of thirst in this study was not due to a generalized depression of motivated behaviors or to the induction of sleep (Gvilia et al., 2005; McKinley et al., 2015), as refeeding after food deprivation was unaffected by VGAT neuron stimulation. Thus, GABAergic MnPO neurons are unlikely to limit drinking by responding to presystemic signals from gastric distension, which should influence both drinking and eating (Stricker and Stricker, 2011). But they may respond to other sensory stimuli, such as cool water on the tongue, or to signaling associated with hypervolemia, hypertension, and/or hypotonicity (i.e., systemic inhibition of thirst).

Our findings complement a recent study that demonstrated marked water consumption during optogenetic stimulation of the CaMKII/neuronal nitric oxide synthase (nNOS)-expressing (presumably glutamatergic) SFO neurons, and reductions in water consumption during brief stimulation of SFO GABAergic neurons (McKinley et al., 2015; Oka et al., 2015). The MnPO is a primary efferent target of the SFO (Miselis, 1981; Swanson and Lind, 1986; Oka et al., 2015). Furthermore, the MnPO has much more extensive downstream projections to the dorsomedial nucleus, lateral hypothalamus, paraventricular nucleus of the thalamus, and periaqueductal gray matter than the SFO (Saper and Levisohn, 1983; Swanson and Lind, 1986); hence, glutamatergic MnPO neurons may be a critical intermediary in SFO-driven drinking responses, as described by Oka et al. (2015). Furthermore, as we show that MnPO GABAergic neurons have an intense input to the SFO, they may inhibit CaMKII/nNOS-expressing SFO neurons that promote drinking during dehydration, thus explaining the large reduction in water consumption we observed in dehydrated mice in the present study.

In conclusion, we have shown that $\mathrm{MnPO} / \mathrm{OVLT}$ glutamatergic neurons promote water consumption, while MnPO GABAergic neurons inhibit water consumption. Our anatomical results suggest that GABAergic and glutamatergic MnPO/OVLT neurons bidirectionally influence water consumption; it will be important in future studies to determine whether they have similar dichotomous control of vasopressin release, and possibly other aspects of the autonomic, neuroendocrine, and behavioral responses to perturbations in water/electrolyte homeostasis. The roles played by these counterpoised populations of neurons in the MnPO/OVLT in the regulation of other functions, such as sleep or body temperature, also remain important areas for study. In addition, determining whether some neurons influence multiple diverse functions, or whether there are functionally differentiated, and presumably genetically differentiated, populations of MnPO neurons specializing in specific responses, will be critical in understanding how the controls of these functions are interrelated.

\section{References}

Bourque CW (2008) Central mechanisms of osmosensation and systemic osmoregulation. Nat Rev Neurosci 9:519-531. CrossRef Medline

Buggy J, Johnson AK (1977) Anteroventral third ventricle periventricular ablation: temporary adipsia and persisting thirst deficits. Neurosci Lett 5:177-182. CrossRef Medline

Cunningham JT, Sullivan MJ, Edwards GL, Farinpour R, Beltz TG, Johnson AK (1991) Dissociation of experimentally induced drinking behavior by ibotenate injection into the median preoptic nucleus. Brain Res 554: 153-158. CrossRef Medline

Cunningham JT, Beltz T, Johnson RF, Johnson AK (1992) The effects of ibotenate lesions of the median preoptic nucleus on experimentallyinduced and circadian drinking behavior in rats. Brain Res 580:325-330. CrossRef Medline

De Luca LA Jr, Xu Z, Schoorlemmer GH, Thunhorst RL, Beltz TG, Menani JV, Johnson AK (2002) Water deprivation-induced sodium appetite: humoral and cardiovascular mediators and immediate early genes. Am J Physiol Regul Integr Comp Physiol 282:R552-R559. CrossRef Medline

Grob M, Trottier JF, Drolet G, Mouginot D (2003) Characterization of the neurochemical content of neuronal populations of the lamina terminalis activated by acute hydromineral challenge. Neuroscience 122:247-257. CrossRef Medline

Gvilia I, Angara C, McGinty D, Szymusiak R (2005) Different neuronal populations of the rat median preoptic nucleus express C-Fos during sleep and in response to hypertonic saline or angiotensin-II. J Physiol 569:587-599. CrossRef Medline

Hayar A, Bryant JL, Boughter JD, Heck DH (2006) A low-cost solution to measure mouse licking in an electrophysiological setup with a standard analog-to-digital converter. J Neurosci Methods 153:203-207. CrossRef Medline

Kinsman B, Cowles J, Lay J, Simmonds SS, Browning KN, Stocker SD (2014) Osmoregulatory thirst in mice lacking the transient receptor potential vanilloid type 1 (Trpv1) and/or type 4 (Trpv4) receptor. Am J Physiol Regul Integr Comp Physiol 307:R1092-R1100. CrossRef Medline

Krashes MJ, Shah BP, Madara JC, Olson DP, Strochlic DE, Garfield AS, Vong L, Pei H, Watabe-Uchida M, Uchida N, Liberles SD, Lowell BB (2014) An excitatory paraventricular nucleus to AGRP neuron circuit that drives hunger. Nature 507:238-242. CrossRef Medline

Lazarus M, Yoshida K, Coppari R, Bass CE, Mochizuki T, Lowell BB, Saper CB (2007) EP3 prostaglandin receptors in the median preoptic nucleus are critical for fever responses. Nat Neurosci 10:1131-1133. CrossRef Medline

Mangiapane ML, Thrasher TN, Keil LC, Simpson JB, Ganong WF (1983) Deficits in drinking and vasopressin secretion after lesions of the nucleus medianus. Neuroendocrinology 37:73-77. Medline

McKinley MJ, Hards DK, Oldfield BJ (1994) Identification of neural pathways activated in dehydrated rats by means of Fos-immunohistochemistry and neural tracing. Brain Res 653:305-314. CrossRef Medline

McKinley MJ, Walker LL, Alexiou T, Allen AM, Campbell DJ, Di Nicolantonio R, Oldfield BJ, Denton DA (2008) Osmoregulatory fluid intake but not hypovolemic thirst is intact in mice lacking angiotensin. Am J Physiol Regul Integr Comp Physiol 294:R1533-R1543. CrossRef Medline

McKinley MJ, Yao ST, Uschakov A, Mcallen RM, Rundgren M, Martelli D (2015) The median preoptic nucleus: front and centre for the regulation of body fluid, sodium, temperature, sleep and cardiovascular homeostasis. Acta Physiol (Oxf) 214:8-32. CrossRef Medline

Miselis RR (1981) The efferent projections of the subfornical organ of the rat: a circumventricular organ within a neural network subserving waterbalance. Brain Res 230:1-23. CrossRef Medline

Nakamura K, Morrison SF (2008) A thermosensory pathway that controls body temperature. Nat Neurosci 11:62-71. CrossRef Medline

Oka Y, Ye M, Zuker CS (2015) Thirst driving and suppressing signals encoded by distinct neural populations in the brain. Nature 520:349-352. CrossRef Medline

Oldfield BJ, Bicknell RJ, McAllen RM, Weisinger RS, McKinley MJ (1991) Intravenous hypertonic saline induces Fos immunoreactivity in neurons throughout the lamina terminalis. Brain Res 561:151-156. CrossRef Medline

Paxinos G, Franklin KB (2012) The mouse brain in stereotaxic coordinates. New York: Elsevier.

Prager-Khoutorsky M, Bourque CW (2015) Anatomical organization of the rat organum vasculosum laminae terminalis. Am J Physiol Regul Integr Comp Physiol 309:R324-R337. CrossRef Medline

Renaud LP, Cunningham JT, Nissen R, Yang CR (1993) Electrophysiology of central pathways controlling release of neurohypophysial hormones. Focus on the lamina terminalis and diagonal band inputs to the supraoptic nucleus. Ann N Y Acad Sci 689:122-132. CrossRef Medline

Robertson GL (1991) Disorders of thirst. London: Springer.

Saper CB, Levisohn D (1983) Afferent connections of the median preoptic nucleus in the rat: anatomical evidence for a cardiovascular integrative mechanism in the anteroventral third ventricular (Av3v) region. Brain Res 288:21-31. CrossRef Medline 
Simpson JB, Routtenberg A (1973) Subfornical organ: site of drinking elicitation by angiotensin II. Science 181:1172-1175. CrossRef Medline

Stocker SD, Toney GM (2005) Median preoptic neurones projecting to the hypothalamic paraventricular nucleus respond to osmotic, circulating Ang II and baroreceptor input in the rat. J Physiol London 568:599-615. CrossRef Medline

Stricker EM, Stricker ML (2011) Pre-systemic controls of fluid intake and vasopressin secretion. Physiol Behav 103:86-88. CrossRef Medline

Swanson LW, Lind RW (1986) Neural projections subserving the initiation of a specific motivated behavior in the rat: new projections from the subfornical organ. Brain Res 379:399-403. CrossRef Medline

Szymusiak R, McGinty D (2008) Hypothalamic regulation of sleep and arousal. Ann N Y Acad Sci 1129:275-286. CrossRef Medline

Taylor AC, McCarthy JJ, Stocker SD (2008) Mice lacking the transient receptor vanilloid potential 1 channel display normal thirst responses and central Fos activation to hypernatremia. Am J Physiol Regul Integr Comp Physiol 294:R1285-R1293. CrossRef Medline

Thrasher TN, Keil LC, Ramsay DJ (1982) Lesions of the organum vasculosum of the lamina terminalis (OVLT) attenuate osmotically-induced drinking and vasopressin secretion in the dog. Endocrinology 110: 1837-1839. CrossRef Medline

Thunhorst RL, Xu Z, Cicha MZ, Zardetto-Smith AM, Johnson AK (1998) Fos expression in rat brain during depletion-induced thirst and salt appetite. Am J Physiol 274:R1807-R1814. Medline

Uschakov A, Gong H, McGinty D, Szymusiak R (2007) Efferent projections from the median preoptic nucleus to sleep- and arousal-regulatory nuclei in the rat brain. Neuroscience 150:104-120. CrossRef Medline

Vong L, Ye C, Yang Z, Choi B, Chua S Jr, Lowell BB (2011) Leptin action on GABAergic neurons prevents obesity and reduces inhibitory tone to POMC neurons. Neuron 71:142-154. CrossRef Medline

Yang CR, Senatorov VV, Renaud LP (1994) Organum vasculosum lamina terminalis-evoked postsynaptic responses in rat supraoptic neurones in vitro. J Physiol 477:59-74. CrossRef Medline

Zardetto-Smith AM, Thunhorst RL, Cicha MZ, Johnson AK (1993) Afferent signaling and forebrain mechanisms in the behavioral control of extracellular fluid volume. Ann N Y Acad Sci 689:161-176. CrossRef Medline 